\title{
TITLE:
}

\section{$<$ Note> Use of Leaf-sponge and Leaf-spoon by Juvenile Chimpanzees at Mahale}

\section{$\operatorname{AUTHOR}(S)$ :}

Matsusaka, Takahisa; Kutsukake, Nobuyuki

\section{CITATION:}

Matsusaka, Takahisa ...[et al]. < Note> Use of Leaf-sponge and Leafspoon by Juvenile Chimpanzees at Mahale. Pan Africa News 2002, 9(1): 6-9

ISSUE DATE:

2002-06

URL:

http://hdl.handle.net/2433/143405

\section{RIGHT:}

Copyright (C) Pan Africa News. 
came to the site of a tree-hollow at 15:29. At first, she drank water directly by mouth. Then she broke off a large twig of Grewia sp., put it into the hollow, and licked several times. Then she put one drenched leaf of the twig into her mouth, sucked it, and started to use it as a leaf-sponge; she repeated "soak and suck" actions to drink water. At 15:32 Athena (4 yrs female) came and solicited J idda to play. They left the hollow.

After 2 minutes, Athena returned alone to the hollow and drank water by hand. Then Athena picked up a leaf-sponge from the hollow, which apparently was the same as that used by J idda, and used it twice to drink water. Then she broke off a twig and drank water with it. She left the site at 15:37.

Case 2: On 25 J anuary, J idda came to the site where Xmas ( 5 yrs male) had drunk water in a treehollow directly by mouth at 14:49. Jidda found another tree-hollow $3 \mathrm{~m}$ away from Xmas and al so drank water by mouth. After 2 minutes, $\mathrm{J}$ idda tore off a vine and drank water with it repeatedly. Xmas left at 14:52. He never used vines or leaves to drink. J idda kept on drinking by mouth and hand. At 14:53, J idda picked a nearby leaf of Saba comorensis, squeezed it into the hollow, and used it repeatedly as a leaf-sponge to drink (F igure 1). Once, she failed to put the sponge into the hollow and dropped it. She picked another leaf of Saba immediately, squeezed it into the hollow, and again used it as a leaf-sponge. After she dropped the sponge again, $J$ idda drank water by hand for a while and left the hollow at 15:01. She went to the hollow from which Xmas had drunk water, looked into it, and put her hand inside. However, she left there without drinking water.

Case 3 : On 1 February, Athena came to

\section{Introduction}

Use of a leaf-sponge to drink water in a tree-hollow is commonly observed in most long-term study sites of wild chimpanzees $(1,2)$. Use of a leaf-spoon was sporadically reported at Gombe (3) and Bossou $(4,5)$. At Mahale, use of drinking tools was rare (6). However, we found several cases of using leaves to drink water at Mahale during a field study from Sept. 2000 to Sept. 2001. All attempts were performed by juveniles. We report details of these observations.

\section{Use of Leaf-sponge}

Case 1: On 25 November, J idda (5 yrs female) water in a tree-hollow by hand at 15:27. After a minute Aqua left. Athena picked a vine and drank water with it, but she threw it away at once. She then drank water by hand repeatedly. At 15:31, Athena picked some leaves, squeezed them into the hollow, and used them repeatedly to drink water as a leaf-sponge. At 15:37, Athena picked several other leaves, squeezed them into the hollow, and again used them as a leaf-sponge. From 15:39, Athena used vines to drink water and left the hollow at 15:43. 

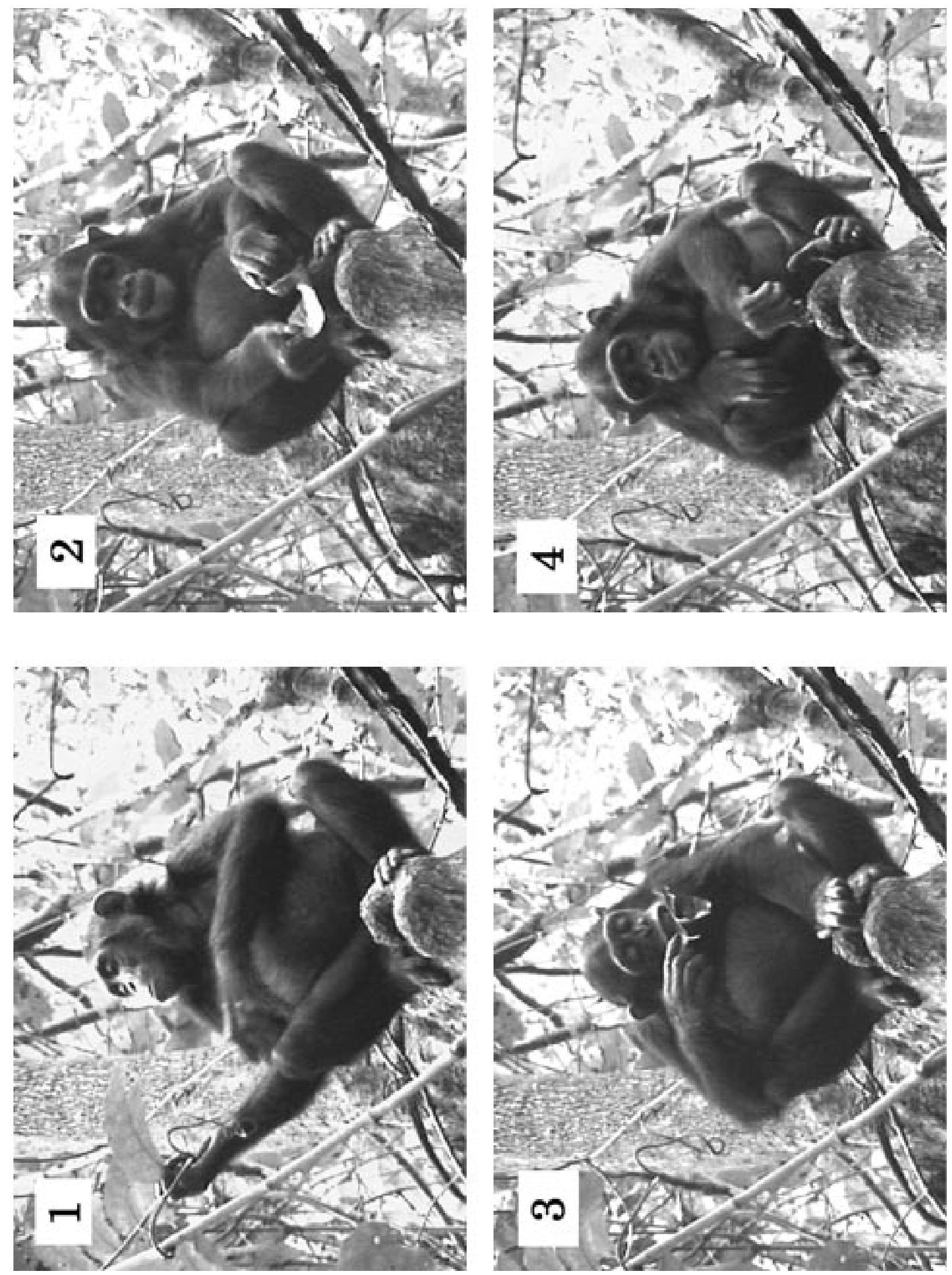

Figure 1. Typical sequence of drinking water with leaf-sponge ( $\mathrm{idda}$ on $25 \mathrm{~J}$ anuary). (1) Picking a leaf. (In all instances, within $1 \mathrm{~m}$ from the hollow.) (2) Squeezing the uncrumpled leaf into the hollow. (3) Picking up the soaked leaf and stuffing it into the mouth and sucking water. (4)Taking the crumpled leaf out of the mouth and again putting it into the hollow. Then repeating "soak and suck" actions. 


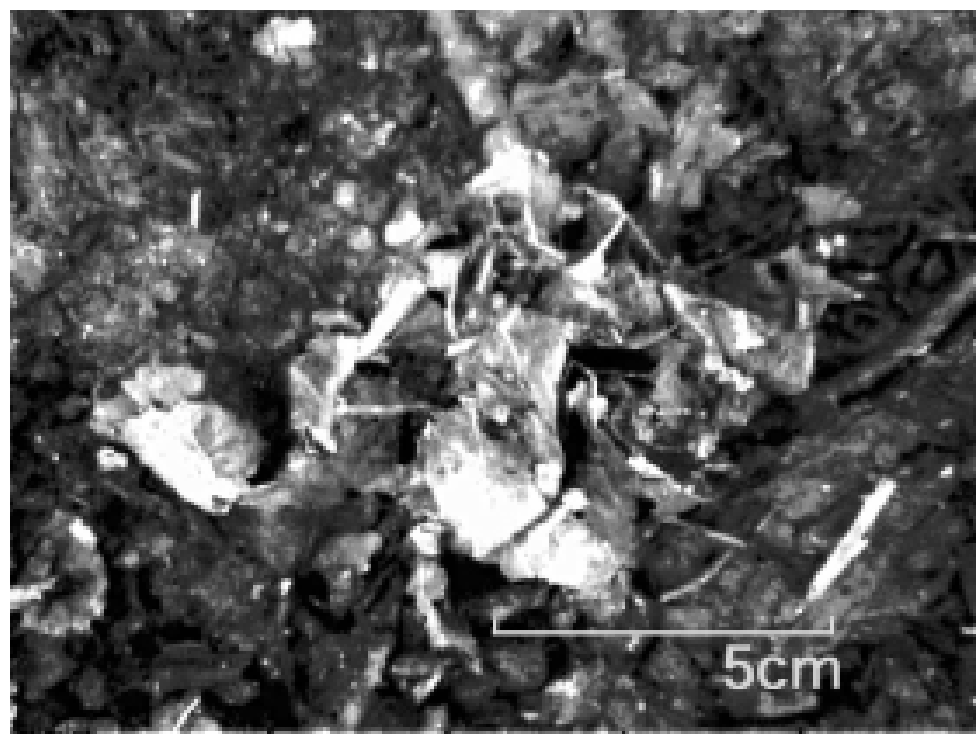

Figure 2. A leaf-sponge used by Jidda on $25 \mathrm{~J}$ anuary (Saba comorensis).

At 15:49, J idda came to the hollow, picked leafy-twig, and squeezed it into the hollow. After picking it out, J idda put one drenched leaf of the twig into her mouth and started to use it as a leaf-sponge. Once, J idda picked a leaf of Cordia millenii, stirred the hollow with its leafstalk, and then picked up the sponge with her hand. It was assumed that she used the leafstalk to pick out the sponge stuck in the hollow. At 16:00, J idda lay down and it became hard to observe what she did. J idda departed at 16:07.

Matsusaka recorded all these cases with a digital video camera, and Figure 1 shows a typical sequence of using a leaf-sponge.

\section{Use of Leaf-spoon}

On 31 May, J idda and Xmas started playing by a stream at 8:57. At 9:06, J idda picked up a leaf (4 $\mathrm{cm}$ by $9 \mathrm{~cm}$ ) from the ground, immersed it into the stream, and brought it to her mouth for pouring water. While drinking, J idda flicked the leaf several times with her fingers so that she could drink the water drops on it. Also, J idda repeatedly splashed the water surface of the stream with her fingers and mouth. Xmas observed her behavior and started playing with $J$ idda again. After 3 minutes, Xmas picked up a dead leaf $(7 \mathrm{~cm}$ by $14 \mathrm{~cm})$ from the ground and used this as a leaf-spoon to drink water as J idda did (9:21). Also, Xmas splashed the water surface with his hands. They left at 10:00.

\section{Discussion}

Although use of a leaf-sponge is not an established culture of chimpanzees at Mahale, two juveniles used leaf-sponges in a relatively natural way. Moreover, their ways of processing leaves were different from those observed at other study sites; Jidda and Athena first squeezed an uncrumpled leaf into the hollow in most instances; however, at Gombe and Bossou, chimpanzees first stuff leaves into the mouth and make them crumpled before they soak them in water $(3,5)$. This led us to assume that these techniques may be transmitted by imitation, at least at Gombe and Bossou. A tool-use to pick out a sponge, apparently stuck in a hollow, was also observed once, which indicates high problem-solving skills and innovativeness of the juvenile.

Use of a leaf-spoon was also found. However, we suspect that the main purpose in the present case was not drinking water, since they could easily do this without using a leaf. Rather, it is reasonable to assume that these acts were performed as part of playing.

It is worth noting that behavioral patterns were instantly imitated by juveniles, who have great behavioral flexibility, a factor that may be the fundamental mechanism causing the present cultural diversity among wild chimpanzee study sites $(1,2)$. Considering the rarity of observations of using drinking tools by Mahale chimpanzees previous to this study, it would be valuable to confirm whether the present juveniles continue using drinking tools when they become adults or cease using leaves as they grow up.

This study was financially supported by the Monbusho Scientific Research Fund (Basic Research A1, \#12375003 to T. Nishida) and J SPS Research Fellowships for Young Scientists (to NK).

\section{References}

(1) Whiten A, Goodall J , McGrew WC, Nishida T, Reynolds V, Sugiyama Y, Tutin CEG, Wrangham RW, Boesch C 1999. Cultures in chimpanzees. Nature 399: 682-685.

(2) Whiten A, Goodall J , McGrew WC, Nishida T, Reynolds V, Sugiyama Y, Tutin CEG, 
Wrangham RW, Boesch C 2001. Charting cultural variation in chimpanzees. Behaviour. 138: 1481-1516.

(3) Goodall J 1986. The chimpanzees of Gombe. Belknap Press, Cambridge, Mass.

(4) Sugiyama $Y$ 1995. Drinking tools of wild dhimpanzees at Bossou. Am. J. Primatol. 37: 263-269.

(5) Tonooka R 2001. Leaf-folding behavior for drinking water by wild chimpanzees (Pan troglodytes verus) at Bossou, Guinea. Animal Cognition 4: 325-334.

(6) Nishida T 1990. A quarter century of research in the Mahale Mountains: an overview. In: The chimpanzees of the Mahale Mountains, Nishida T (ed), Univ. of Tokyo Press, Tokyo, pp. 3-35. 